\title{
The Exploring Research on Resource Mapping Method for Virtual Network Deployment
}

\author{
Jie Qian ${ }^{1, a}$, Hanxi Li ${ }^{1, b}$, Yanping Wang ${ }^{1, c}$ \\ ${ }^{1}$ China Tobacco Zhejiang Industrial Co., LTD, Hangzhou, 310009, China \\ a qianj@zjtobacco.com; ${ }^{b}$ lihx@zjtobacco.com; ' wangyp@zjtobacco.com
}

Keywords: Virtual Network; Resource Mapping; Hierarchical Management

\begin{abstract}
. virtualization technology is an important means to achieve the current cloud computing environments to maximize resources utilization, how to effectively use technology in virtual networks within the data center is based on the key challenges of virtual network resource allocation problem faced. In this thesis, a virtual network and virtual resources management agent Agent-based virtualization management proposes a centralized hierarchical tree network architecture virtualized environment resources management, and designs and implements building virtual experimental network hierarchy in the network test platform Resource mapping framework. Virtual network mapping is more than one kind of resource constraints, the optimization process much conflicting goals. Virtual network mapping strategy and algorithm research traitor has important theoretical and practical value.
\end{abstract}

\section{Introduction}

Irrational resource allocation not only makes the resource utilization is low, and sometimes even difficult to meet the needs of a dynamic resource applications. Virtualization technology is an important means to achieve the current cloud computing environments to maximize resources utilization, but the existing resource allocation to virtual machines mainly granularity, lack of effective management of network resources, making it difficult to meet for sensitive network resources Quality of Service application needs [1-2]. Network resources over the quality of service for cloud applications have a greater impact. However, the existing allocation of resources to the virtual machine as the main mode of granularity, the lack of effective management of network resources, it is difficult to ensure that the above application requirements for network resources. For this reason, by the user application abstract virtual network and virtual network to achieve network-based virtualization of resources is effectively a way to solve this problem [3].

Introduced virtual network on the basis of physical network virtualization concept, you can build multiple virtual networks on the same physical network, to meet the individual needs of the application at the same time improve the utilization of network resources. How to effectively use the virtual network technology within the data center, based on the virtual network is an important challenge facing the problem of resource allocation [4]. This thesis proposes a hierarchical tree structure under centralized network resource management in virtualized environments based virtualization management of virtual networks and virtual resource management agent proxy, then mapping problem when building and running a virtual network from a virtual network between Dynamic Resource assign two virtual network resource management aspects of the study, final design and implement hierarchical resource mapping framework to build virtual experimental network test platform on the network. .

\section{Virtual network resource mapping analysis}

Network virtualization recently in academia and industry are subject to considerable attention as an important solution to overcome the shortcomings of the current Internet. Network virtualization enables complex virtual network (VN) running on the underlying network abstraction and isolation (SN) while the substrate network resources [5-6]. As described in Figure 1, a virtual network 
consists of a group (such as a virtual node virtual router), reside in different virtual nodes and links interconnecting substrate, the substrate by a physical path network.

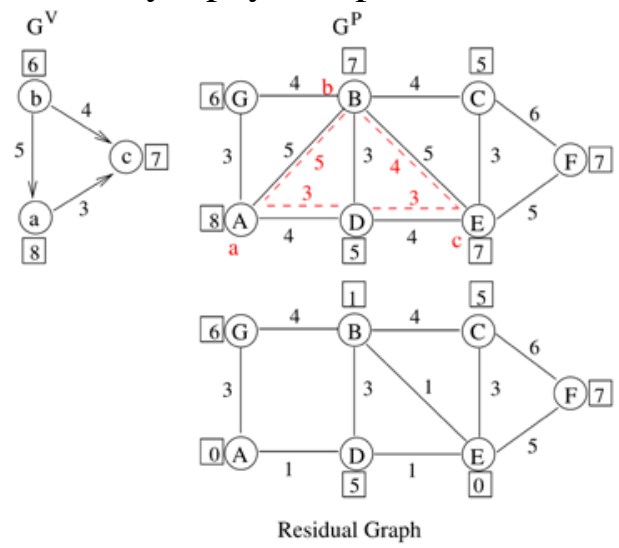

Figure 1: Example of virtual network mapping

Network virtualization aims to make the virtual network a subset of the substrate as a specialized network resources to provide network services to VN users. Network virtualization offers great potential to support innovative new technologies and architectures essentially change the Internet. The most important issue is the use of network virtualization underlying network resources effectively provided in Figure 1. Effective methods and algorithms for mapping virtual network mapping VNs substrate. Good virtual network mapping algorithm will help to improve resource utilization and load balancing substrate. Virtual network embedding / allocation shown in Figure 2 is the choice of the substrate nodes have sufficient CPU and memory as well as the substrate path has sufficient bandwidth, while minimizing the use of the total resources of the substrate. However, VN embedded problems, limitations of virtual nodes and virtual links are difficult np problem. .

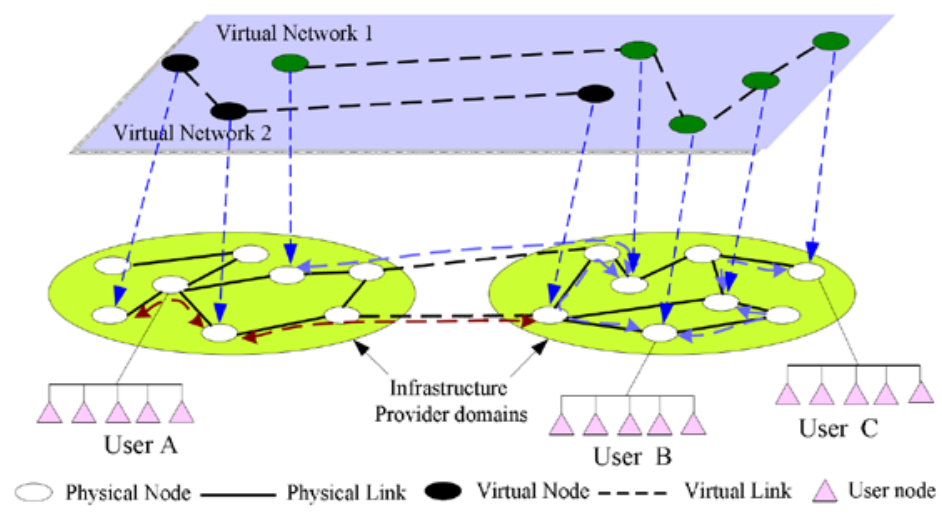

Figure 2. Virtual Network Mapping

So many heuristics and algorithms solutions proposed to solve VN embedding / allocation problems [7]. Precious research VN embedded algorithm in a centralized manner. A central controller is responsible for receiving and distributing VN request a set of virtual resources to build a virtual network. The controller maintains all status information about the underlying network, so it can get the optimal solution for embedded virtual network. However, to maintain the global status information throughout the matrix will be a major challenge in the underlying physical network of large-scale, highly dynamic. Therefore, based on multi-agent distributed virtual network embedding algorithm method way of cooperation between the substrates allocate virtual resource node. Dispersion algorithms rely on a large number of messages transmitted between the substrate nodes to implement virtual network embedding. Great to underlying network communication overhead to additional load. But the lack of a global solution is not the best view of the entire underlying network. In addition, heuristic, custom and greedy proposals it does not exist to resolve how they propose VN mapping algorithm design and implementation of mapping framework, mechanisms and protocols. Propose a regulatory framework to balance the scalability and performance overhead of centralized and decentralized algorithms. Architecture uses geographic information layering and multi-domain structure coupled with virtual nodes to better control and management of the substrate 
from the global network of resources. And design elements to make a separate virtual network management embedding parallel processing, fault management and resource scheduling to meet online $\mathrm{VN}$ requests.

\section{Hierarchical management framework}

Future virtual resource management of the network to provide specific QOS VN network-based virtualization is a key role to ensure efficient utilization of network resources and users. Resource management solutions is currently divided into two types: centralized and decentralized algorithms. Centralized approach may have serious limitations scalability and decision-making efficiency mapping VNs when the substrate is a highly dynamic environment. And dispersing more efficient mapping algorithm VN requests in a distributed way, but it is more complicated to achieve is due to message exchange and protocol design. It is to use a layered approach coupled weigh $\mathrm{VN}$ decomposition. In our management framework, the substrate is subdivided into several management areas (zoning method is beyond the scope of this article), each region has a management element (I) is responsible for monitoring the implementation of network management functions and virtual resource allocation. MEs all areas of management constitute the hierarchy of the first layer. In addition, there is a control system (CS) is responsible for resource scheduling and accounting management across domains.

CS can be considered the second level of the hierarchy to control and manage the MEs. Upon receiving a request $\mathrm{VN}$, CS will be divided into sub-virtual $\mathrm{VN}$ request network (sub-VNs) and sending sub-VNs domain mapped to the appropriate management. In our framework, each image I maintain the local network topology and capacity available virtual resources. And CS communicates with the MEs obtain the state of the substrate. Hierarchy feature allows the frame to get good scalability. In addition, the centralized controller need to map the request as a whole unit VN valuable studies $(10,12)$, but the multi-domain architectures mapping feature requests so that each parallel multiple VN VN my map, and improve efficiency. In this section, the working principle of hierarchical management framework we describe a virtual network mapping, shown in Figure 3.

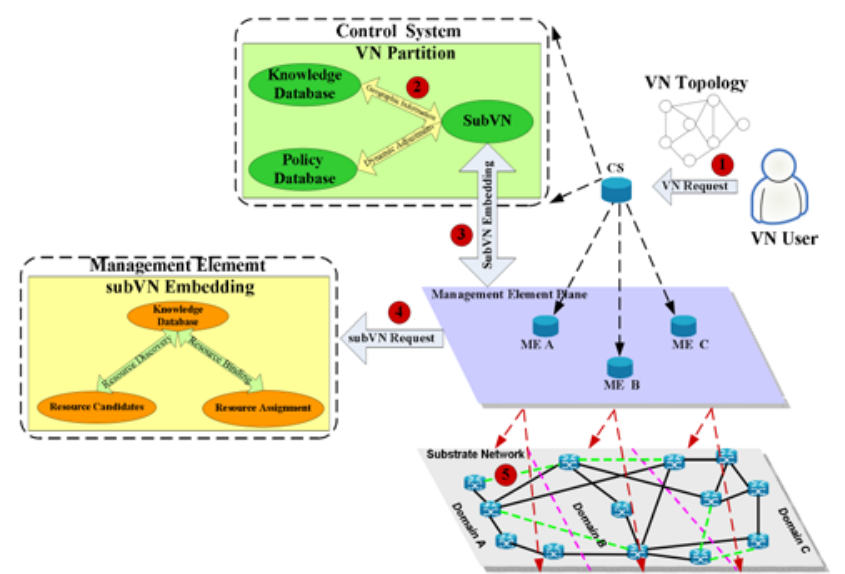

Figure 3. Hierarchical Management Framework of VN Mapping

VN requests the user's hand CS (step 1). CS first partition VN request for the location information of several sub-VNs virtual node (step 2), then the sub-VN sends the request to the appropriate (step 3). I use optimization algorithms available Select the virtual resources to be used a substrate, and then set the sub-VNs, namely virtual nodes and virtual link sub-VNs (step 4). After sub-VNs instantiated each management domain, Virtual Connect sub-VNs by the c to achieve a global view of the substrate (step 5) Set between. Throughout the virtual network mapping process, $\mathrm{CS}$ is only responsible for the contact $\mathrm{VN}$ requests and set up virtual management domains. MEs are committed to sub-VNs embedding. This feature an exploded view, in a hierarchical manner, a plurality of substrates VN topology effectively and efficiently. . 


\section{Resource scheduling}

Our framework is more flexible and multi-level domain structure arrange physical resources hosted virtual resources. VN request arrives when there are a lot in a short time, some of the VN request is rejected or postponed due to the resources of the substrate is limited. However, in our hierarchical management framework, a virtual resource shortages can be resolved by the resource scheduling management domains between adjacent. CS coordinates a global view of the requester and the substrate knowledge repository and other neighborhood management arrangements resources to meet the needs of the requester virtual resources. If I were to respond sub-VN request requires a lot of resources at a certain time, but the domain cannot support this request, so the request to send a message to the CS virtual resources. Upon receipt by the news, CS First, check out the status information to select a neighborhood B (domain resources to meet the demand temporarily transfer the process shown in Figure 4 resource scheduling.

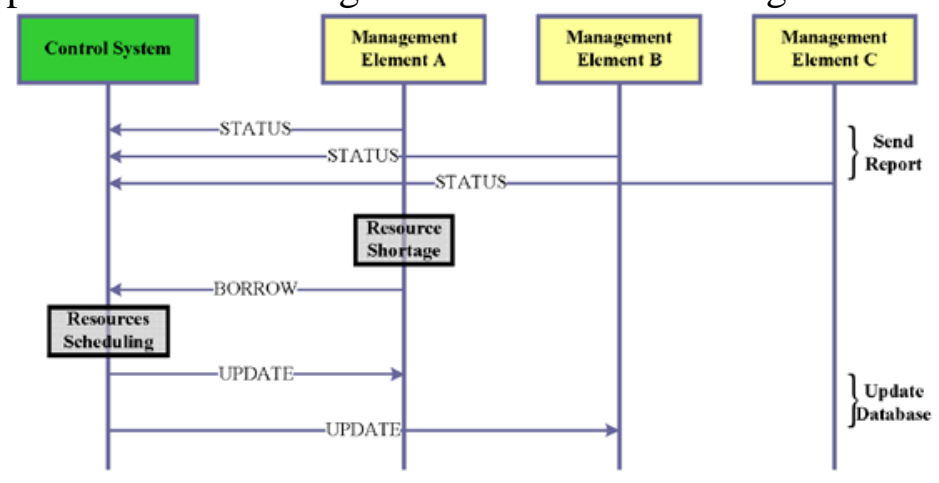

Figure 4. The schematic diagram of Resource Scheduling

Temporary transfer of resources the duration of sub-VN request lifecycle. When life sub-VN requested period, the borrowed resources in another domain should give back to his master. CS is responsible for restoring the original borrowers and lenders topology information. However, this is not free resource scheduling, resource rents accounting operation in the field of records management by computer processing.

\section{Conclusion}

Irrational resource allocation not only makes the resource utilization is low, and sometimes even difficult to meet the needs of a dynamic resource applications. Lack of resource allocation based on existing virtual machines for the size of the effective management of network resources, network resources makes it difficult to meet the demand for quality of service-sensitive applications. To do this, the user application to build a virtual network and virtual network based on the allocation of resources is seen as an effective way to solve this puzzle. In this paper, in order to maximize resource utilization as a target for a cloud environment based on resource allocation virtual network launched an in-depth study. In this thesis, a virtual network and virtual resources management agent Agent-based virtualization management proposes a centralized hierarchical tree network architecture virtualized environment resources management, and designs and implements building virtual experimental network hierarchy in the network test platform Resource mapping framework.

\section{Reference}

[1] George A P, De R. Review of temporal bone dissection teaching: how it was, is and will be[J]. The Journal of Laryngology \& Otology, 2010, 124(02): 119-125.

[2] Schlick T. Molecular modeling and simulation: an interdisciplinary guide: an interdisciplinary guide[M]. Springer Science \& Business Media, 2010.

[3] Kataoka R, Ebisuzaki T, Kusano K, et al. Three - dimensional MHD modeling of the solar wind structures associated with 13 December 2006 coronal mass ejection[J]. Journal of Geophysical Research: Space Physics (1978-2012), 2009, 114(A10). 
[4] Tajima K, Chen K K, Takahashi N, et al. Three-dimensional finite element modeling from CT images of tooth and its validation[J]. Dental materials journal, 2009, 28(2): 219-226.

[5] Ahmed K, Keeling A N, Fakhry M, et al. Role of virtual reality simulation in teaching and assessing technical skills in endovascular intervention[J]. Journal of Vascular and Interventional Radiology, 2010, 21(1): 55-66.

[6] Xiang Y, Arora J S, Abdel-Malek K. Physics-based modeling and simulation of human walking: a review of optimization-based and other approaches[J]. Structural and Multidisciplinary Optimization, 2010, 42(1): 1-23.

[7] Waran V, Narayanan V, Karuppiah R, et al. Injecting realism in surgical training-initial simulation experience with custom 3D models[J]. Journal of surgical education, 2014, 71(2): 193-197. 